\title{
Odor-Based Nanomechanical Discrimination of Fuel Oils Using a Single Type of Designed Nanoparticles with Nonlinear Viscoelasticity
}

Kota Shiba,* Gaku Imamura, and Genki Yoshikawa

Cite This: ACS Omega 2021, 6, 23389-23398

Read Online

S1 Supporting Information

ABSTRACT: Odors are one of the most diverse and complicated gaseous mixtures so that their discrimination is challenging yet attractive because of the rich information about their origin. The more similar the properties of odors are, the more difficult the discrimination becomes. The practical applications, however, often demand such discrimination, especially with a compact sensing platform. In this paper, we show that a nanomaterial designed for a specific type of odors can clearly discriminate them even with a single nanomechanical sensing channel. Fuel oils and their mixture are used as a model target that has similar chemical properties but different compositions mainly consisting of paraffinic, olefinic, naphthenic, and

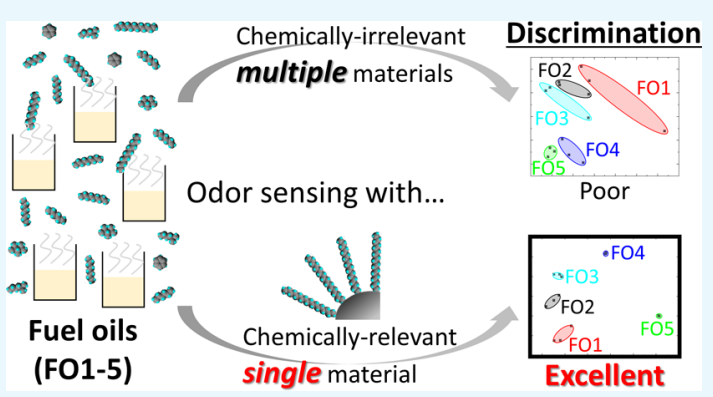
aromatic hydrocarbons. We demonstrate using octadecyl functionalized silica-titania nanoparticles that the difference in the compositions is successfully picked up based on their high affinity for the aliphatic hydrocarbons and alkyl chain length dependent nonlinear viscoelastic behavior. Such a properly designed material is proved to derive sufficient information from a series of analytes to discriminate them even with a single sensing element. This approach provides a guideline to prepare various sensors whose response properties are distinct and optimized depending on applications.

\section{INTRODUCTION}

++ Discrimination of odors is one of the major challenges in analytical science and related fields. An odor is often composed of tens of, hundreds of, or even thousands of components, making it extremely diverse and complicated. Thus, the discrimination always requires organized techniques. ${ }^{1}$ Gas chromatography-mass spectrometry has been a well-known conventional technique for decades and is utilized as a standard in the world, although it suffers from the requirements of a skillful operator and a rather long measurement time. Under the circumstances, a sensor array concept emerges to discriminate odors with much easier operation, shorter measurement time, less cost, and more compact setup. ${ }^{2-9}$ The array consists of multiple channels whose materials/coatings are different so that each channel responds uniquely to a variety of odors. On the one hand, this approach works to discriminate odors with different properties and/or compositions. But on the other hand, to discriminate odors with rather similar characteristics, a random line-up of functional channels does not work efficiently. Some synergistic, weakened, or neutralized effects between the components of odors make the discrimination even more difficult. $^{10-12}$ For these reasons, sensors and each of their channels at least need to be properly designed to somehow pick up any slight difference between such similar species.

Fuel oils including gasoline, kerosene, and diesel are mainly composed of hydrocarbons, meaning that these oils have similar chemical properties. Furthermore, there is a serious worldwide problem that some illegal adulteration frequently happens by mixing a certain amount of impurity to a fuel oil to increase its volume. ${ }^{13,14}$ Gas chromatography-mass spectrometry ${ }^{15,16}$ and other spectroscopic techniques such as infrared spectroscopy, ${ }^{17-20}$ Raman spectroscopy, ${ }^{18,21-23}$ ion mobility spectroscopy, ${ }^{24}$ terahertz spectroscopy, ${ }^{25-27}$ and ultraviolet spectrosco$\mathrm{py}^{28}$ have contributed to analyzing fuel oils, although they have several drawbacks mentioned earlier that limit the quick and easy test. Alternatively, sensor arrays are more suitable for such a purpose because of their potential easiness, compactness, and inexpensiveness. Several previous studies have reported that sensor arrays consisting of metal-oxide-based semiconductors, ${ }^{29-31}$ quartz crystal microbalances coupled with capacitance transducers, ${ }^{32}$ functional Au nanoparticles, ${ }^{33}$ various polydiacetylenes, $^{34}$ and dye-silica/alumina mixtures ${ }^{35}$ are utilized to discriminate the fuel oils. Although even tens of different channels are aligned for discrimination in some cases, only a few of them seem to provide useful information. Importantly, the hydrocarbons existing in the fuel oils are indeed diverse; they mainly include paraffinic, olefinic, naphthenic, and aromatic

Received: June 22, 2021

Accepted: July 23, 2021

Published: August 27, 2021 
hydrocarbons with hundreds of structural isomers whose number of carbon atoms ranges from $\mathrm{C} 4$ to $\mathrm{C} 40 .{ }^{16,36,37}$ Therefore, what we have to consider here is how to derive measurable differences from these various mixtures consisting of such chemically similar species whose slight difference in composition matters. On the basis of our previous studies, ${ }^{38,39}$ one prerequisite is to design a sensor to have a roughly similar property-hydrophobicity, in this case-with that of the fuel oils so that the sensor becomes sensitive to them. To detect a minute difference between the fuel oils, a sensor needs to have sensitivity sufficient for obtaining a clear sensing signal to extract effective features. The other key is to design the sensor surface to derive further detailed information that reflects such difference based on some common features in all components. As aliphatic hydrocarbons are major components of fuel oils, alkyl chain length would be one of the targets for this purpose. The successful acquisition of alkyl chain length-related information and its efficient transduction into sensor responses would realize precise discrimination of fuel oils even with a single sensor channel, leading to a practical compact sensing system.

In this paper, we describe an effective combination of a nanomechanical sensor and a nanomaterial with nonlinear viscoelasticity that enables us to clearly discriminate fuel oils. We accomplish this by designing a functional nanoparticle that is composed of octadecyl modified silica-titania hybrid nanoparticles (C18-STNPs) ${ }^{38,39}$ and using them as a gas sensing material for a Membrane-type Surface stress Sensor (MSS). ${ }^{40}$ As compared to the other types of conventional sensors, MSS is free from the following common problems: high temperature operation, limited options for a sensing material, vibration/air flow induced instability, cross talk between channels, bulky setup, insufficient sensitivity, and high power consumption. ${ }^{40-42}$ The single-channel sensor functionalized with C18-STNPs is chemically/physically stable, is specifically sensitive to hydrocarbons that are one of the major components of the fuel oils, provides distinct resolution on their adsorption/desorption processes based on the change in the viscoelastic behavior of C18-STNPs, and thus responds to the fuel oils differently depending on their components. We demonstrate how the present approach works by measuring regular gasoline, premium gasoline, kerosene, diesel, and $10 \%$ kerosene dissolved in regular.

\section{EXPERIMENTAL SECTION}

Chemicals. Octadecyltriethoxysilane (ODTES; Tokyo Chemical Industry Co., Ltd., >85.0\%), dodecyltriethoxysilane (DDTES; Tokyo Chemical Industry Co., Ltd., >95.0\%), titanium tetraisopropoxide (TTIP; Tokyo Chemical Industry Co., Ltd., purity N/A), isopropyl alcohol (IPA; Wako Pure Chemical Industries, Ltd., >99.7\%), aqueous ammonia solution $\left(\mathrm{NH}_{3} \mathrm{aq}\right.$; Kanto Chemical Co., Inc., 28.0-30.0\%), and octadecylamine (ODA; Aldrich, Inc., 97.0\%) were used to prepare nanomaterials. Poly(vinylpyrrolidinone) (PVP; SigmaAldrich, Inc., average molecular weight: 360,000), poly(allylamine hydrochloride) (PAA; Sigma-Aldrich, Inc., average molecular weight: 17,500), and poly(methyl methacrylate) (PMMA; Sigma-Aldrich, Inc., average molecular weight: 15,000) were used as coating materials. Ethanol (EtOH; Wako Pure Chemical Industries, Ltd., >99.5\%) and toluene (Kanto Chemical Co., Inc., >99.5\%) were used to prepare the polymer solutions for spray coating. Fuel oils including regular gasoline, premium gasoline, kerosene, and diesel were purchased from ENEOS Corporation and utilized for their vapor measurements. In addition, the following chemicals were also used for the same purpose: formaldehyde aqueous solution (formaldehyde aq.; Wako Pure Chemical Industries, Ltd., 35$38 \%$ ), acetic acid (Wako Pure Chemical Industries, Ltd., >99.7\%), N,N-dimethylformamide (DMF; Tokyo Chemical Industry Co., Ltd., >99.5\%), acetone (Sigma-Aldrich, Inc., $>99.5 \%$ ), 2-butanone (Wako Pure Chemical Industries, Ltd., >99.0\%), methanol ( $\mathrm{MeOH}$; Kanto Chemical Co., Inc., $>99.8 \%)$, 1-butanol (1-BuOH; Sigma-Aldrich, Inc., >99.5\%), 1-pentanol (1-PeOH; Sigma-Aldrich, Inc., >98.0\%), $n$-hexane (C6; Wako Pure Chemical Industries, Ltd., >96.0\%), $n$-heptane (C7; Nacalai Tesque, Inc., >99.0\%), $n$-octane (C8; Nacalai Tesque, Inc., $>98.0 \%$ ), $n$-nonane (C9; Nacalai Tesque, Inc., $>98.0 \%$ ), $n$-decane (C10; Nacalai Tesque, Inc., >98.0\%), $n$ undecane (C11; Nacalai Tesque, Inc., $>99.0 \%$ ), n-dodecane (C12; Nacalai Tesque, Inc., $>99.0 \%$ ), benzene (Wako Pure Chemical Industries, Ltd., $>99.5 \%$ ), toluene (Wako Pure Chemical Industries, Ltd., >99.5\%), xylene (mixture of $o$-, $m$-, and $p$-xylene; Wako Pure Chemical Industries, Ltd.), 1,2dichlorobenzene (1,2-DCB; Sigma-Aldrich, Inc., >99\%), and 1,3-dichlorobenzene (1,3-DCB; Sigma-Aldrich, Inc., >99\%). All the chemicals were used as received. The chemical structures of ODTES, PVP, PAA, and PMMA are shown in Figure S1.

Nanomaterial Synthesis. C18-STNPs were synthesized by a multistep nucleation-controlled growth method with some minor modifications. ${ }^{38,39,43}$ The procedure is schematically shown in Scheme S1. Briefly, five starting solutions (solutions $\mathrm{A}-\mathrm{E}$ ) were prepared. The detailed composition of each solution is summarized in Table S1. The solutions A, B, C, and D were individually flowed in perfluoroalkoxyalkane tubes (PFA; 1.0 $\mathrm{mm}$ inner diameter, 1/16 in. outer diameter, product of YMC Co., Ltd.) using a syringe pump (CXN1070, product of ISIS, Co., Ltd.) at $10 \mathrm{~mL} / \mathrm{min}$. The solutions $\mathrm{A}$ and $\mathrm{B}$ and solutions $\mathrm{C}$ and $\mathrm{D}$ were mixed, respectively, in a polytetrafluoroethylene (PTFE) fluidic channel with a Y-shape junction (the crosssectional area of the channel: approximately $1 \mathrm{~mm}^{2}$, KeyChem mixer, product of YMC Co., Ltd.). After that, the two resultant reaction solutions-solution $\mathrm{A}+\mathrm{B}$ and solution $\mathrm{C}+\mathrm{D}$-were mixed in the second fluidic channel placed just after the first two fluidic channels. The first and second fluidic channels were connected with $100 \mathrm{~mm}$ PFA tubes. Then, the mixture of the four solutions (A-D) was flowed through a PFA tube with a length of $700 \mathrm{~mm}$ and added into solution $\mathrm{E}$ under magnetic stirring. After the addition, the final reaction solution was aged at room temperature for a week without magnetic stirring.

Spray Coating Procedure. The suspension of C18-STNPs was spray-coated onto the surface of MSS by using a spray coater (rCoater, product of Asahi Sunac Co.). MSS consisted of a thin silicon membrane $(2.8 \mu \mathrm{m}$ thick, $300 \mu \mathrm{m}$ in diameter) suspended by four beams where piezoresistors are embedded. ${ }^{40}$ When the coating adsorbed the analytes, it swelled and generated surface stress that was detected with the piezoresistors. As we had four membranes on a chip, the spray coating procedure was applied to each membrane one by one using a mask. To prepare the suspension, C18-STNPs were centrifuged at $9000 \mathrm{rpm}$ for $10 \mathrm{~min}$. The sediment was carefully washed with IPA several times followed by the addition of the IPA/water mixture ( $\mathrm{vol} / \mathrm{vol}=3 / 5)$ into it. The concentration was set at approximately $1 \mathrm{~g} / \mathrm{L}$. Before spray-coating, sonication was applied to get C18-STNPs dispersed as much as possible (some aggregates might be still recognized). Then, the suspension was loaded in a syringe and flowed through a PTFE tube at $3 \mathrm{~mL} /$ min by using a syringe pump (YSP-201, product of YMC Co., 
Ltd.). The suspension was introduced into a spray nozzle and sprayed with the help of two types of carrier air (atomizing air: $0.030 \mathrm{MPa}$ and patterning air: $0.030 \mathrm{MPa}$ ) to form homogeneous droplets. An MSS chip was mounted on a stage that was heated at approximately $100{ }^{\circ} \mathrm{C}$ to quickly evaporate the droplets. The stage moved back and forth, while the spray nozzle moved from the left to the right at $15 \mathrm{~mm} / \mathrm{s}$ with a 0.3 $\mathrm{mm}$ pitch. The distance between the spray nozzle and stage was set at $100 \mathrm{~mm}$. The coating process was repeated 20 times to get the coating with a thickness of around $1.5 \mu \mathrm{m}$ (determined with the Dektak-XT Stylus Profiler (DXT-A), Bruker Corp.).

A similar protocol was performed for three polymers: PVP, PAA, and PMMA. As they dissolved in different solvents, $\mathrm{EtOH}$, water/EtOH mixture ( $\mathrm{vol} / \mathrm{vol}=1 / 4)$, and toluene were utilized, respectively. Their concentration was set at $5 \mathrm{~g} / \mathrm{L}$. Optical microscope images of all the four coatings are shown in Figure 1
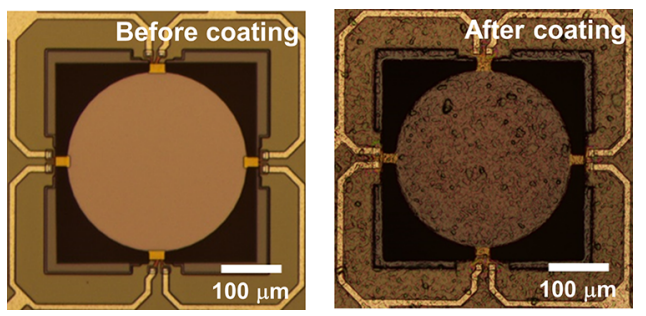

Figure 1. Optical microscope images of MSS before and after being coated with C18-STNPs.

and Figure S2. Since a spray nozzle was going back and forth multiple times above MSS during a coating cycle, the whole sensor surface was eventually coated with each material. According to a previous report, ${ }^{44}$ MSS is sufficiently robust against the inhomogeneity of the coating owing to its symmetric structure. The signal deviation was reported to be in the range of only 5-6\% even with a receptor layer having a coffee ring structure, which is difficult to control. Since the receptor layers prepared in the present study using a spray coater were rather uniform without a distinct feature, the present coating could provide even less deviation in the sensing signals.

Vapor Measurement. We measured vapors of 28 samples including 5 fuel oils and their mixture (10\% kerosene dissolved in regular) and 23 chemicals including ultrapure water, formaldehyde aq., acetic acid, DMF, 2 ketones (acetone and 2-butanone), 5 alcohols ( $\mathrm{MeOH}, \mathrm{EtOH}, \mathrm{IPA}, 1-\mathrm{BuOH}$, and 1$\mathrm{PeOH}$ ), 7 alkanes (C6 to $\mathrm{C} 12$ ), and 5 aromatic compounds (benzene, toluene, xylene, 1,2-DCB, and 1,3-DCB). These samples were measured using the following experimental setup. A schematic of the setup is shown in Scheme S2. The C18STNPs-coated MSS chip was placed in a chamber with its interior volume in the range of microliters, and the chamber was carefully sealed with O-rings. Two mass flow controllers (MFC1 and MFC2; FCST1005C-4F2-F100N2, purchased from Fujikin Inc.) were utilized to introduce nitrogen into the chamber at a flow rate of $100 \mathrm{~mL} / \mathrm{min}$. MFC1 was for purging to accelerate the desorption of adsorbents, and MFC2 was for introducing a sample vapor together with nitrogen as a carrier. Both injection and purging were performed for $30 \mathrm{~s}$. and this cycle was repeated four times for all the samples. The details of the injectionpurging cycle and corresponding sensor output are shown in Figure S3. In this study, $1 \mathrm{~mL}$ of the sample liquid was added into a small vial capped with a rubber lid, and two needles connected to PTFE tubes were stuck into the headspace of the vial through the rubber lid. One end of the PTFE was connected to MFC2 and the other end of the PTFE tube was connected to a vacant vial to make the mixed gas sample homogeneous. Another PTFE tube stuck into the mixing vial was connected to the chamber. MFC1 and the vacant vial were set in the same manner and connected to the mixing vial as well. The two MFCs were switched every $30 \mathrm{~s}$ to conduct a sample injection-purging cycle, which was repeated four times. The data were recorded at a bridge voltage of $-0.5 \mathrm{~V}$ and a sampling rate of $20 \mathrm{~Hz}$. The data collection program was designed by LabVIEW (NI Corp.). All the experiments were conducted under ambient conditions without any temperature/humidity control.

Principal Component Analysis (PCA). The following four parameters were extracted from an MSS response as features for PCA:

$$
\begin{aligned}
& \text { Parameter 1: }(b-a) /\left(t_{b}-t_{a}\right) \\
& \text { Parameter 2: }(d-c) /\left(t_{d}-t_{c}\right) \\
& \text { Parameter 3: }(f-e) /\left(t_{f}-t_{e}\right) \\
& \text { Parameter 4: }(d-g)
\end{aligned}
$$

where $a, b, c, d, e, f, g, t_{a}, t_{b}, t_{c}, t_{d}, t_{e}, t_{f}$, and $t_{g}$ are denoted in Figure 2. Specifically, $t_{a}, t_{b}, t_{c}, t_{d}, t_{e}, t_{f}$, and $t_{g}$ were defined as $t_{a}=t_{0}+1[\mathrm{~s}]$,

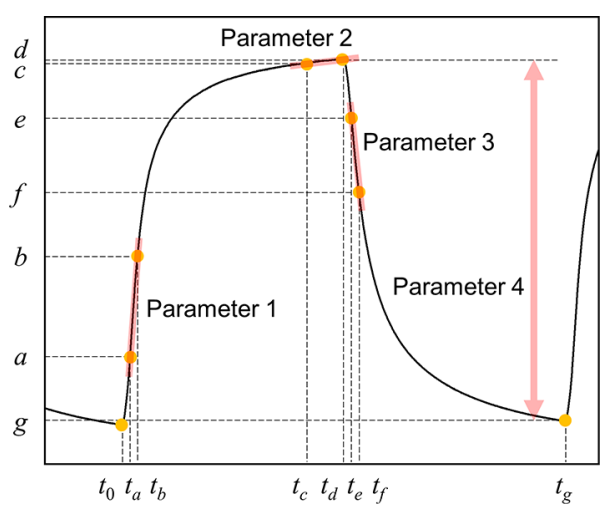

Figure 2. A schematic diagram of the four parameters extracted from a response curve.

$t_{b}=t_{0}+2[\mathrm{~s}], t_{c}=t_{0}+25[\mathrm{~s}], t_{d}=t_{0}+30[\mathrm{~s}], t_{e}=t_{0}+31[\mathrm{~s}], t_{f}=t_{0}$ $+32[\mathrm{~s}]$, and $t_{g}=t_{0}+60[\mathrm{~s}]$, respectively. The responses from the second to fourth injection-purging cycle were used for the feature extraction because the first cycle was usually affected by preadsorbed water molecules and/or residual compounds from previous measurements, resulting in a transient response. Thus, we used 90,150 , and 210 as $t_{0}$. Three sets of the four parameters were extracted accordingly for PCA. The present PCA was performed using Origin Pro 2020 (Lightstone Corp.). These parameters reflect several physicochemical characteristics included in a response: adsorption, quasi-equilibrium, desorption, and adsorption capacity. ${ }^{38,39}$

Material/Coating Characterization. Scanning electron microscope (SEM) images were obtained using a Hitachi Ultrahigh Resolution Scanning Electron Microscope SU8000 at an accelerating voltage of $10 \mathrm{kV}$. Prior to each observation, samples were coated with a few nanometers of platinum.

A particle size distribution was measured by a dynamic light scattering (DLS) technique using an ELSZ-2000 (Otsuka Electronics Co., Ltd.). C18-STNPs were dispersed in trichloromethane and then used for the measurement. 

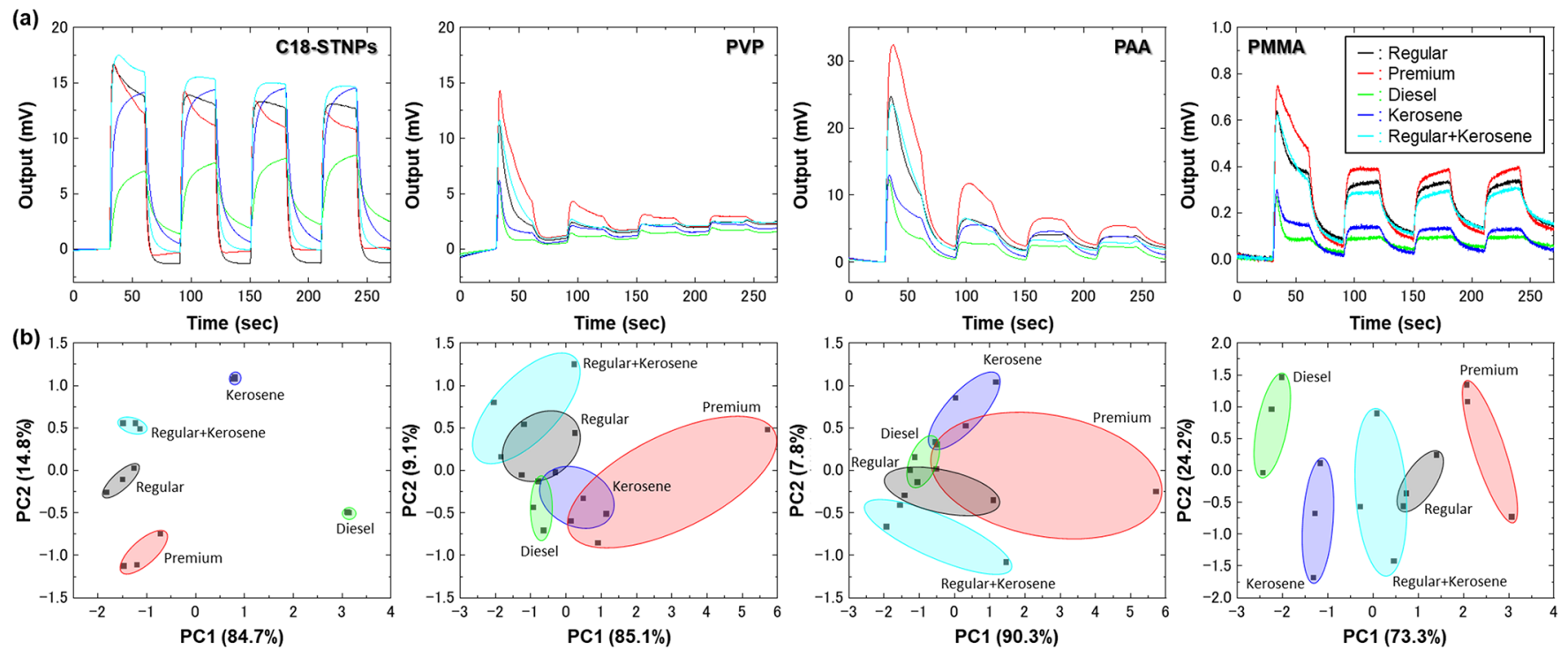

Figure 3. (a) MSS responses to regular (black), premium (red), diesel (green), kerosene (blue), and 10\% kerosene in regular (pale blue). Each panel from the left to the right shows the response measured by the MSS coated with C18-STNPs, PVP, PAA, and PMMA, respectively. (b) Corresponding PCA plots.

Fourier Transform infrared (FT-IR) spectra were measured using a Nicolet 4700 FT-IR spectrometer (Thermo Fisher Scientific Inc.) at a resolution of $2.0 \mathrm{~cm}^{-1}$ and in the range from 4000 to $500 \mathrm{~cm}^{-1}$. The sample powder was homogeneously mixed with $\mathrm{KBr}$, and then the mixture was pressed to form a $\mathrm{KBr}$ disk for the transmission measurements.

Thermogravimetric-differential thermal analysis (TG-DTA) curves were recorded on an SII EXSTAR 6000 TG/DTA6300 at a heating rate of $10{ }^{\circ} \mathrm{C} / \mathrm{min}$ under air flow. $\alpha$-Alumina powder was used as a reference material to obtain DTA curves.

Optical microscope images were taken using an Eclipse $\mathrm{Ni}$ (Nikon Instruments Inc.).

\section{RESULTS AND DISCUSSION}

When the fuel oils including regular gasoline, premium gasoline, kerosene, and diesel are measured with the C18-STNPs-coated MSS (denoted as C18-MSS), all the responses show very different shapes and output intensity as summarized in Figure 3a. As expected, the four fuel oils are discriminated by PCA as shown in Figure $3 \mathrm{~b}$ because the four features used here are defined to correlate with the profile of the responses. These results mean that the single sensor channel sufficiently works to discriminate odors that originate from samples even with very similar chemical properties. We find that regular gasoline containing $10 \%$ of kerosene gives a response that is not same as that of either gasoline or kerosene. Thus, the corresponding points in the PCA plot are also distinct. They locate between those of pure regular and pure kerosene but are rather close to regular, most probably reflecting the ratio of the mixture. Estimation of the ratio of regular and kerosene could be possible because predicting the concentration of each component in a water-methanol-ethanol ternary mixture is possible by means of machine learning. ${ }^{38}$ In contrast, three polymers including PVP, PAA, and PMMA, which are frequently coated on various sensor arrays, ${ }^{45-47}$ result in much smaller responses with similar shapes for all samples. Note that the remarkably large first curve is due to a temporary mixing of the analyte molecules with preadsorbed water molecules in the receptor layer and their desorption induced by the introduction of analytes. Con- sequently, the PCA plots for these polymers exhibit some overlaps among each sample, meaning that the samples are not well-discriminated. Although using 12 features extracted from the three polymers simultaneously provides better discrimination, the plots are still broadly scattered as shown in Figure S4.

Importantly, unlike the three polymers, C18-STNPs also show distinct responses to a series of alkanes consisting of C6 to $\mathrm{C} 12$ as well as a well-separated PCA plot, as shown in Figure $4 a, b$. Although the concentration of these alkanes is different each other because their amount in the carrier gas depends on their vapor pressure, we can still confirm that C18-STNPs are able to differentiate the seven alkanes regardless of the effect of concentration. According to a previous report, features extracted from a decay curve are not significantly affected by concentration. ${ }^{48}$ Thus, we also perform PCA only based on each decay curve and extract the change in their slope over time (see Figure S5 for details). The PCA plot is shown in Figure 4c. The data points for each alkane are still group-wise in spite of the smaller number of features used for the analysis, whereas the polymers show apparent overlap. Considering the fact that each fuel oil mainly contains hydrocarbons with different carbon numbers, the robust property to derive any information that relates to the difference between structurally similar molecules should be a major reason why the fuel oils are successfully discriminated only by C18-STNPs. This result is similar to that observed in chromatography measurements using a column modified with a nonpolar phase such as methyl polysiloxane. ${ }^{49,50}$

One of the most important aspects is the viscoelastic behavior of the C18-STNPs coating. Different from the three polymers, C18-STNPs give symmetric responses in their injection and purging of the analytes except in the first cycle, as shown in Figures 3 and 4. According to the viscoelastic model proposed previously, $^{51}$ a typical nanomechanical sensor coated with a viscoelastic material exhibits symmetric sensing responses for gas injection and carrier gas purge. This behavior reflects two different processes: diffusion of sample gas and stress relaxation. Thus, the symmetric responses indicate that C18-MSS follows the viscoelastic model. In contrast, the response shapes of the 

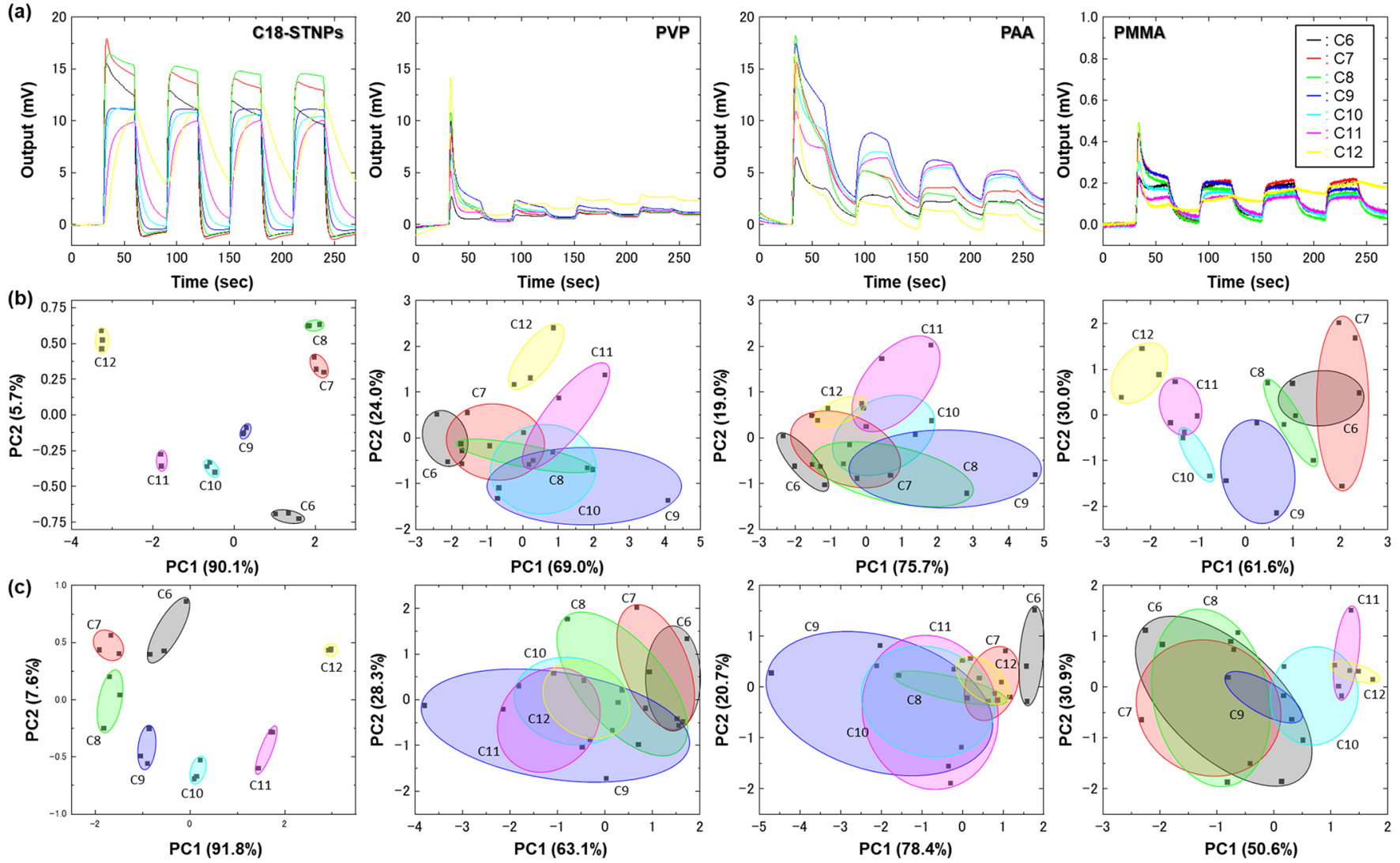

Figure 4. (a) MSS responses to a series of alkanes including C6 (black), C7 (red), C8 (green), C9 (blue), C10 (pale blue), C11 (pink), and C12 (yellow). Each panel from the left to the right shows the response measured by the MSS coated with C18-STNPs, PVP, PAA, and PMMA, respectively. $(b, c)$ Corresponding PCA plots. The ones in (b) were obtained using the four parameters shown in Figure 1, whereas those in (c) were obtained using the three parameters that were extracted only from a decay curve (see Figure S5 for details).
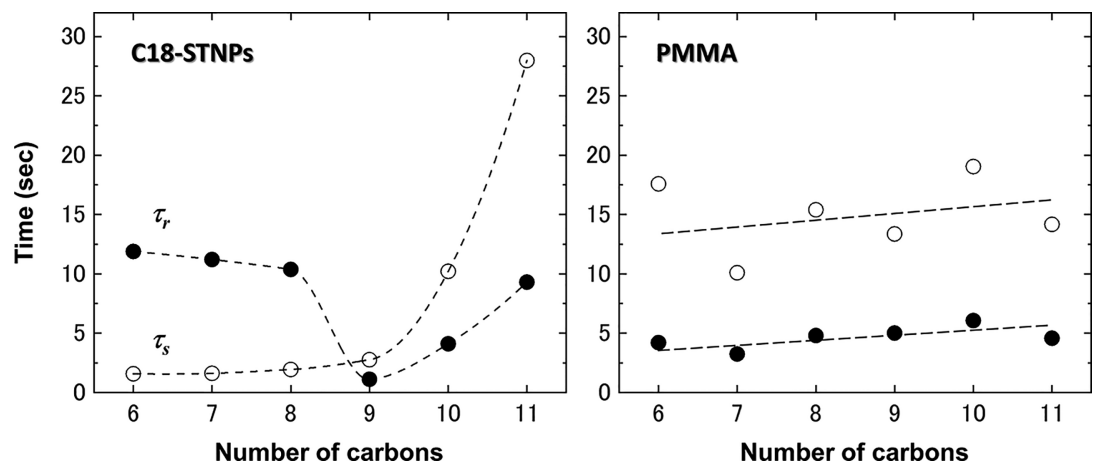

Figure 5. Plots of $\tau_{s}$ (open circle) and $\tau_{r}$ (closed circle) as a function of the number of carbons.

polymers are completely asymmetric; they rise fast and decay much slowly. This asymmetry indicates that the responses of the polymer-coated MSS include irregular processes that the viscoelastic model does not consider. For example, the polymers initially swell by adsorbing analytes, replacing preadsorbed water molecules, and then get soft or even almost dissolved because of the diffusion-induced filling of the analytes in their matrix. Taking into account that the matrix consisting of the polymers has more accessible sites than that consisting of C18-STNPs as the interior of the nanoparticles is not accessible, it takes a much longer time or more cycles to reach a steady state. The transient responses of the polymers would reflect such characteristics. Compared to organic polymers, inorganic-oxide-based materials such as C18-STNPs are chemically and physically more stable.
In addition to their chemical affinity to hydrocarbons discussed already, the chemical/physical robustness of C18-STNPs is also an important feature for the clear discrimination.

For the quantitative discussion on the interaction between the coatings and analytes, we focus on two specific time constants for diffusion and relaxation, $\tau_{s}$ and $\tau_{r}$, respectively. ${ }^{52}$ These constants are estimated by fitting the fourth decay curve of each response in Figure 4 (except C12 because of its insufficient measurement time for reasonable fitting) with an equation given by:

$$
V(t)=V_{0}+A_{1} e^{-\left(t-t_{0}\right) / \tau_{s}}+A_{2} e^{-\left(t-t_{0}\right) / \tau_{r}}
$$

where $V(t)$ is the output voltage, $t$ is the time, $V_{0}$ is the offset value, and $t_{0}$ is the time at which the purging starts, respectively. 


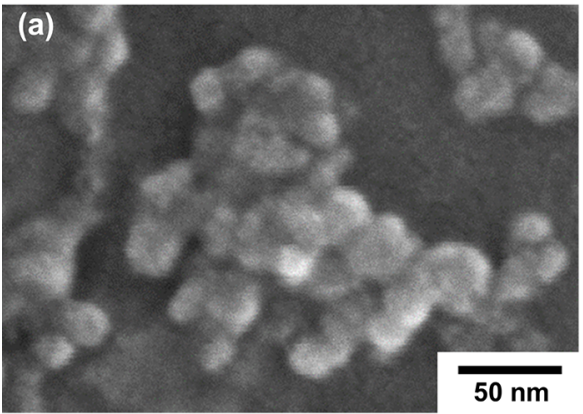

(b)
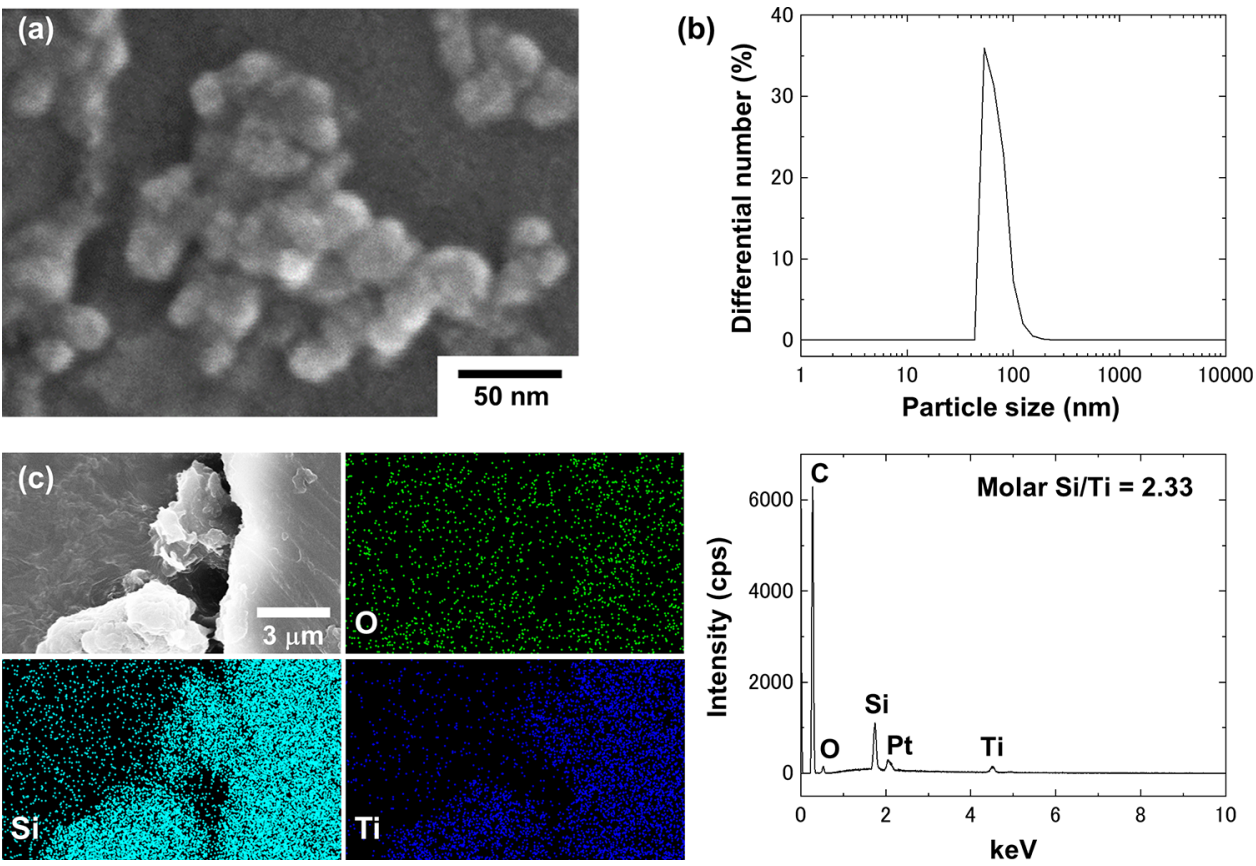

Figure 6. (a) An SEM image of C18-STNPs. (b) A particle size distribution of C18-STNPs dispersed in trichloromethane. (c) An SEM image and corresponding elemental mappings $(\mathrm{O}, \mathrm{Si}$, and $\mathrm{Ti}$ ) of $\mathrm{C} 18$-STNPs. The bottom right panel shows a corresponding energy-dispersive $\mathrm{X}$-ray spectrum.

$A_{1}$ and $A_{2}$ are the fitting parameters that correspond to the amplitude of the sensing signal. Since only irregular responses are available for PVP and PAA, we apply the fitting to C18STNPs and PMMA and obtain $\tau_{s}$ and $\tau_{r}$ as shown in Figure 5. Interestingly, in the case of C18-STNPs, $\tau_{r}$ shows higher values than $\tau_{s}$ from $\mathrm{C} 6$ to $\mathrm{C} 8$ and then steeply decreases to have lower values than $\tau_{s}$ from $\mathrm{C} 9$ to $\mathrm{C} 11 . \tau_{s}$ gradually increases up to $\mathrm{C} 9$ and then shows a steep rise until C11. It is reported that an overshoot/downshoot appears in its response curve when $\tau_{r}$ is higher than $\tau_{s}{ }^{52}$ which is seen in the responses for C6 to C8. As $\tau_{r}$ and $\tau_{s}$ reflect the interaction between C18-STNPs and the alkanes, the obtained trend suggests the presence of a threshold where the interaction between them nonlinearly changes after C8. In contrast, $\tau_{s}$ and $\tau_{r}$ for PMMA are rather monotonic; $\tau_{s}$ is always higher than $\tau_{r}$, and they both increase gradually as the number of carbons increases. Such a monotonic variation of $\tau_{s}$ and $\tau_{r}$ as well as higher $\tau_{s}$ values is consistent with that reported in the literature for some polymers including PMMA. ${ }^{52}$ These totally different behaviors of interacting with a series of alkanes are the dominant factor in the significant difference in the discrimination ability of each vapor. The complicated nonlinear variation of the time constants observed in C18-STNPs allows for the present discrimination even with a single material.

Then, we estimate how much concentration of each alkane can be measured by C18-STNPs. To investigate this point, the alkanes are exposed to a constant flow of nitrogen at 20 to 100 $\mathrm{mL} / \mathrm{min}$ for $300 \mathrm{~s}$, and the exact amount of the alkanes evaporated under the conditions is determined (see Figure S6 for details). Output voltages measured at corresponding conditions are shown in Figure S7. Taking account of the signal-to-noise ratio of MSS with the typical noise level of about $1 \mu \mathrm{V},{ }^{40} \mathrm{C} 18$-STNPs could still get some signal for all the alkanes at a single ppm or sub-ppm concentration, especially single ppb for undecane and dodecane. One of the reasons for such high sensitivity could be due to the high stiffness of C18-STNPs. As nanomechanical sensors tend to show a higher maximum response when a stiffer material is coated on their surface, ${ }^{53}$ it is reasonable that C18-STNPs composed of inorganic constituents whose Young's modulus are typically tens of GPa or higher result in such a high sensitivity, at least compared to any organic polymers whose Young's moduli are typically a few GPa or less. It should be also noted that, for gas/solid equilibrium-based chemical sensors including MSS, the sensitivity depends not on the absolute concentration but on the partial vapor pressure. ${ }^{48,54}$ This behavior is confirmed in Figure S7; the signal intensities of the MSS depend not on the concentration of each vapor that spans from about 19,500 ppm (saturated vapor of hexane at 25 ${ }^{\circ} \mathrm{C}$ ) to $196 \mathrm{ppm}$ (saturated vapor of dodecane at $25^{\circ} \mathrm{C}$ ) but on the flow rate that corresponds to the partial pressure. We also confirm that there is a linearity between the output voltage and concentration of the alkanes except for C6 as shown in Figure $\mathrm{S} 7 \mathrm{~b}$. This linearity seems to hold at least up to tens of ppm range as shown in Figure S8. Considering all the results discussed here, C18-MSS is highly sensitive to alkanes and has very wide measuring range that could be from ppb range to single \% range.

The high sensitivity of C18-STNPs and its response trend discussed earlier are useful to discriminate the fuel oils. Previous studies report that a major difference in the composition of gasoline, kerosene, and diesel stems from the presence and distribution of hydrocarbons whose number of carbons is different each other: $\mathrm{C} 5$ to $\mathrm{C} 15$ for gasoline, C8 to $\mathrm{C} 16$ for kerosene, and $\mathrm{C} 15$ to $\mathrm{C} 50$ for diesel. ${ }^{37}$ With respect to the difference between regular and premium, there are a few critical points to be focused on: ${ }^{16,55}$ the presence/relative amount of 2,2,4-, 2,3,4-, and 2,3,3-trimethylpentane ( $\mathrm{C} 8 \mathrm{H} 18)$; the relative amount of 2- and 3-methylhexane ( $\mathrm{C} 7 \mathrm{H} 16)$; and the total amount of aromatic hydrocarbons. Regular gasoline contains more amount of methylhexanes than premium but contains less or almost no trimethylpentanes. Also, regular gasoline contains less amount of aromatic hydrocarbons than premium, indicating higher volatility in the end. These facts are consistent with the results shown in Figures 3 and 4. Specifically, hydrocarbons with shorter alkyl chains show a sharper initial rise followed by a steeper decay, whereas those with longer alkyl chains result in 

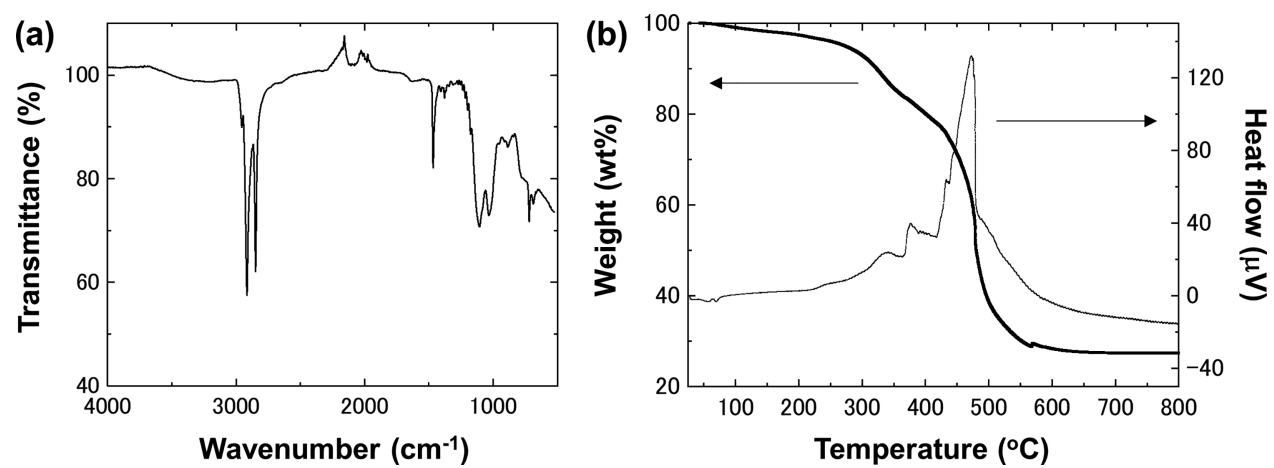

Figure 7. (a) An FT-IR spectrum and (b) TG-DTA curves of C18-STNPs.

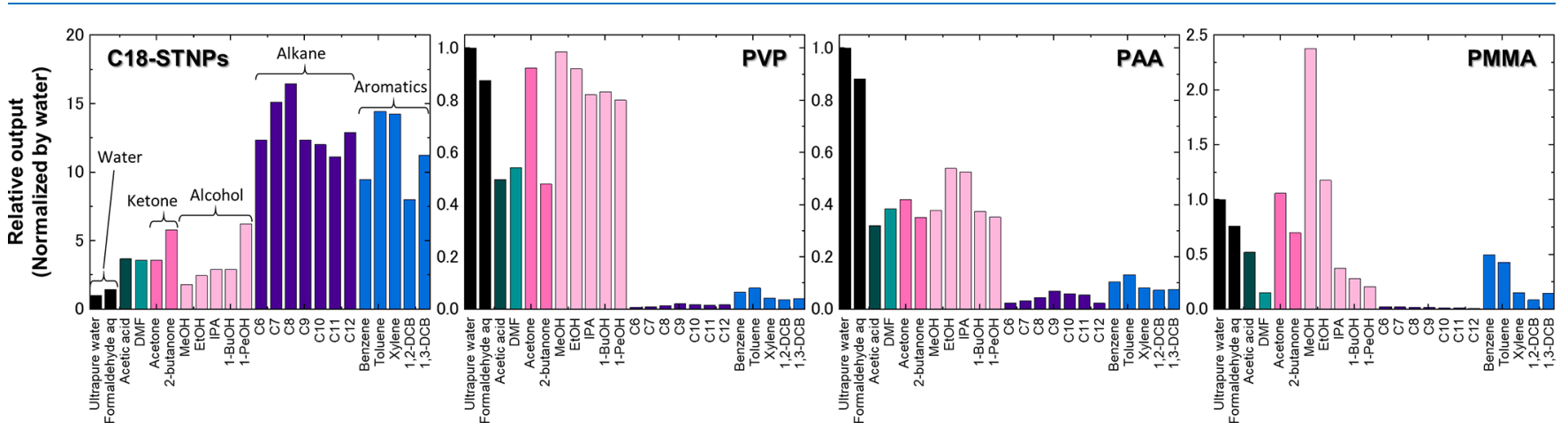

Figure 8. Response trend of C18-STNPs, PVP, PAA, and PMMA to the 23 vapors. The output value for each vapor is extracted from the fourth injection-purging cycle of a corresponding response shown in Figures S9-S12 and then is divided by that for water to normalize.

the opposite trend. Premium gasoline is basically similar to regular gasoline in terms of its rough composition, especially paraffinic hydrocarbons, compared to kerosene or diesel but contains more amount of aromatic hydrocarbons than regular, leading to less entire volatility. Thus, the response for premium shows the initial sharp rise but a smaller response heightparameter 4-than that of regular. Kerosene displays a steeper decay than diesel owing to its components with smaller carbon numbers that should desorb much quicker.

To elucidate the reason for the present clear discrimination from the viewpoint of material, we describe details about C18STNPs. An SEM observation reveals that the size of C18-STNPs is several tens of $\mathrm{nm}$ as seen in Figure 6a. No significant discrepancy is recognized between the SEM-based size and the average size measured by DLS in Figure $6 \mathrm{~b}$. In addition, the DLS measurement shows that C18-STNPs have a narrow particle size distribution. Then, we perform an elemental analysis and confirm that C18-STNPs are composed of silicon, titanium, and oxygen. These elements are distributed homogeneously without aggregation, as shown in Figure 6c. The elemental mapping also allows us to estimate the molar $\mathrm{Si} / \mathrm{Ti}$ ratio to be 2.33 , which is almost consistent with that calculated based on the amount of ODTES and TTIP used for the synthesis-2.67. These results indicate that the hydrolysis and co-condensation reaction of ODTES and TTIP proceed quantitatively to form C18-STNPs.

The presence of octadecyl groups is directly confirmed by the measurement of an FT-IR spectrum that is shown in Figure 7a. Two intense absorption bands are seen at around 2916 and $2848 \mathrm{~cm}^{-1}$. They are ascribed to $\mathrm{C}-\mathrm{H}$ stretching vibrations of the octadecyl groups. Other bands at around 1400, 1100, and $700 \mathrm{~cm}^{-1}$ are all characteristic of octadecyl modified silica. ${ }^{56}$ The actual amount of the octadecyl groups on the surface of C18STNPs is determined by TG-DTA that is presented in Figure $7 \mathrm{~b}$.
The change of the weight as a function of the temperature gives the information. Specifically, the change starting from 150 to $600{ }^{\circ} \mathrm{C}$ accompanying exothermic reactions is attributed to the decomposition of organic species-octadecyl groups, in other words. The weight loss is approximately $70 \mathrm{wt} \%$. It suggests that the yield of C18-STNPs amounts to $97.5 \%$, meaning that the reaction for the synthesis is almost completed. Considering all the characterization results, there are no products that are solely formed from either ODTES or TTIP, and every single particle is composed of titania and silica modified with octadecyl groups whose amount is consistent with that of ODTES used for the synthesis.

In addition to the seven alkanes, we also examine the responses of C18-STNPs to a variety of vapors (raw data are available in Figure S9), as summarized in Figure 8. Obviously, C18-STNPs have a higher affinity for alkanes and aromatic compounds, especially alkanes, than for others. This trend is expected from the structure of C18-STNPs whose entire surface is covered with octadecyl groups. In contrast, PVP-, PAA-, and PMMA-coated MSS show a relatively hydrophilic nature that is indicated by their superior responses to water and other species with a small number of carbons (raw data are available in Figures S10-S12). These response trends of each material are also the major reason why the fuel oils are clearly discriminated only by C18-STNPs.

Although we discriminate the fuel oils sufficiently using a single sensor properly functionalized for the purpose, a higher sensitivity and/or resolution might be required to discriminate samples whose compositions are more slightly different. Toward such a challenge, dodecyl functionalized silica-titania nanoparticles (C12-STNPs) are also synthesized. They respond to the 23 vapors similarly with C18-STNPs as shown in Figure S13. The response trend normalized by water is found to be different; 
the response to alkanes is almost half of C18-STNPs, while the response to aromatics is superior to C18-STNPs (see Figure S14 for details). Judging by these results, functionalization with an alkyl chain longer than C18 might lead to a more distinct discrimination than that shown in Figure $3 \mathrm{~b}$ because kerosene and diesel contain more hydrocarbons with a longer alkyl chain than regular and premium. ${ }^{37}$ Taking advantage of the coimmobilization of multiple functional groups on a single material, ${ }^{38,57}$ further fine-tuning could be possible for a better discrimination. By contrast, although the polymers used in this work do not discriminate the fuel oils well, careful selection of other polymers whose chemical structure is relevant to that of some characteristic molecules included in the fuel oils would bring different results. For example, the combination of hydrophobic nanoparticles and polymers has been reported to work effectively for quantifying the alcohol content of various liquors based on their odors using machine learning. ${ }^{39}$ We note that there is an interesting example of how to diversify the performance of a single material in a different way. Specifically, a QCM-D-based approach has been reported to utilize a single material as multiple sensing channels. ${ }^{58}$ What is demonstrated is that the change in the harmonic number in the channel leads to the modulation of the penetration depth of the shear waves, allowing one to get a specific pattern for a number of analytes at each harmonic number. Such a technique would help derive additional potential of a material. If a form factor of a sensing system allows, the implementation of multiple channels designed for responding differently to the target analytes could certainly enhance the discrimination capability. In addition, advanced approaches in data analysis could realize a more precise and insightful discrimination.

\section{CONCLUSIONS}

We demonstrate that fuel oils including regular gasoline, premium gasoline, kerosene, diesel, and $10 \%$ kerosene dissolved in regular are clearly discriminated using a nanomechanical sensor coated with a single nanomaterial-octadecyl functionalized silica-titania nanoparticles (C18-STNPs). The reason for such a clear discrimination is due to their chemical/physical properties where a series of alkanes are discriminated with high sensitivity potentially in sub-ppm level as well as their distinct, reproducible, and viscoelastic response to each alkane. As C18STNPs demonstrate here, a precisely functionalized material on the basis of its chemical/physical properties and the interacting behavior with target analytes is expected to work sufficiently even with a single channel. It should be noted here that the presented sensor has already achieved the practical discrimination performance (discriminating at least the $10 \%$ kerosene in regular) as well as the practical specifications (small sensor size $\left(<0.1 \mathrm{~mm}^{2}\right)$, room temperature operation (working at a much lower temperature than the ignition temperatures (approximately $200^{\circ} \mathrm{C}$ ) of fuel oils), low power consumption $(<1 \mathrm{~mW})$, mechanical/electrical stability, and quick response $(<1 \mathrm{~min}))$ that are required for detecting the serious problem, fuel adulteration, in the real-world condition.

As demonstrated in this study, it should be important to carefully design each receptor material with a specific purpose from the beginning to develop an effective sensor array, although chemical sensor arrays are usually first built with a set of materials having general chemical/physical divergence. This kind of approach will become more and more critical to discriminate challenging samples such as vapors of various biological fluids, body odors, and breath, in which extremely fine tunings are required to differentiate each sample and those from interfering gases.

\section{ASSOCIATED CONTENT}

\section{SI Supporting Information}

The Supporting Information is available free of charge at https://pubs.acs.org/doi/10.1021/acsomega.1c03270.

Table summarizing the amount of each chemical used to synthesize C18-STNPs; a schematic diagram of the experimental setup for C18-STNP synthesis; a schematic diagram of the experimental setup for vapor measurements; chemical structures of ODTES, PVP, PAA, and PMMA; optical microscope images of MSS coated with PVP, PAA, and PMMA; a plot of the flow rate of two MFCs and output voltage of MSS as a function of time; a PCA plot for five fuel oils measured using PVP, PAA, and PMMA; a schematic diagram of the three parameters extracted from a decay curve; a plot of weight loss for a series of alkanes as a function of flow rate; MSS responses to a series of alkanes and a plot of the output for a series of alkanes as a function of concentration; a plot of output voltage as a function of the concentration of octane; MSS responses to the 23 vapors measured using the $\mathrm{C} 18$ STNP-, PVP-, PAA-, PMMA-, and C12-STNP-coated MSS; and response trend of C18-STNPs and C12-STNPs for the 23 vapors (PDF)

\section{AUTHOR INFORMATION}

\section{Corresponding Author}

Kota Shiba - Center for Functional Sensor \& Actuator (CFSN), National Institute for Materials Science (NIMS), Ibaraki 3050044, Japan; John A. Paulson School of Engineering and Applied Sciences (SEAS), Harvard University, Cambridge, Massachusetts 02138, United States; ○ orcid.org/00000001-7775-0318; Phone: +81-(0)-29-860-4603; Email: SHIBA.Kota@nims.go.jp

\section{Authors}

Gaku Imamura - Center for Functional Sensor \& Actuator (CFSN), National Institute for Materials Science (NIMS), Ibaraki 305-0044, Japan; International Center for Materials Nanoarchitectonics (MANA), National Institute for Materials Science (NIMS), Ibaraki 305-0044, Japan; 이이.org/ 0000-0002-3130-7190

Genki Yoshikawa - Center for Functional Sensor \& Actuator (CFSN), National Institute for Materials Science (NIMS), Ibaraki 305-0044, Japan; Materials Science and Engineering, Graduate School of Pure and Applied Science, University of Tsukuba, Ibaraki 305-8571, Japan; 이 orcid.org/0000-00029136-8964

Complete contact information is available at:

https://pubs.acs.org/10.1021/acsomega.1c03270

\section{Author Contributions}

K.S. conceived the project, designed and performed all the experiments, analyzed the data, and wrote the manuscript. G.I. provided the samples, analyzed the data, discussed the results, and edited the manuscript. G.Y. analyzed the data, discussed the results, and edited the manuscript. All authors agreed with the submission of this work. The manuscript was written through contributions of all authors. 


\section{Notes}

The authors declare the following competing financial interest(s): The authors hold a patent related to the described work (JP 6583836).

\section{ACKNOWLEDGMENTS}

The authors express their sincere gratitude to Ms. Takako Sugiyama (CFSN, NIMS) for all the critical processes from nanoparticle synthesis to characterizations and to Ms. Yuko Kameyama, Ms. Keiko Koda, Ms. Eri Sakon, and Ms. Ikumi Nakakubo (CFSN, NIMS) for the careful coating of the receptor materials and data collection. This work was supported by CFSN, NIMS; JSPS KAKENHI Grant No. JP19KK0141, Grantin-Aid for Scientific Research (A) (No. 18H04168), Grant-inAid for Challenging Research (Pioneering) (No. 20K20554), the Leading Initiative for Excellent Young Researchers, Ministry of Education, Culture, Sports, Science and Technology (MEXT), Japan; The Telecommunications Advancement Foundation, Japan; Marubun Research Promotion Foundation, Japan; Core Research for Evolutional Science and Technology (CREST) (No. JPMJCR1665), Japan Science and Technology Agency (JST); and The Public/Private R\&D Investment Strategic Expansion Program (PRISM), Cabinet Office, Japan.

\section{REFERENCES}

(1) Delahunty, C. M.; Eyres, G.; Dufour, J.-P. Gas chromatographyolfactometry. J. Sep. Sci. 2006, 29, 2107-2125.

(2) Nakamoto, T. Odor sensing system with multi-dimensional data analysis. Jpn. J. Appl. Phys. 2019, 58, 8.

(3) Potyrailo, R. A. Multivariable Sensors for Ubiquitous Monitoring of Gases in the Era of Internet of Things and Industrial Internet. Chem. Rev. 2016, 116, 11877-11923.

(4) Hierlemann, A.; Gutierrez-Osuna, R. Higher-Order Chemical Sensing. Chem. Rev. 2008, 108, 563-613.

(5) Hagleitner, C.; Hierlemann, A.; Lange, D.; Kummer, A.; Kerness, N.; Brand, O.; Baltes, H. Smart single-chip gas sensor microsystem. Nature 2001, 414, 293-296.

(6) Rakow, N. A.; Suslick, K. S. A colorimetric sensor array for odour visualization. Nature 2000, 406, 710-713.

(7) Baller, M. K.; Lang, H. P.; Fritz, J.; Gerber, C.; Gimzewski, J. K.; Drechsler, U.; Rothuizen, H.; Despont, M.; Vettiger, P.; Battiston, F. M.; Ramseyer, J. P.; Fornaro, P.; Meyer, E.; Guntherodt, H. J. A cantilever array-based artificial nose. Ultramicroscopy 2000, 82, 1-9.

(8) Lundström, I.; Erlandsson, R.; Frykman, U.; Hedborg, E.; Spetz, A.; Sundgren, H.; Welin, S.; Winquist, F. Artificial 'olfactory' images from a chemical sensor using a light-pulse technique. Nature 1991, 352, 47-50.

(9) Persaud, K.; Dodd, G. Analysis of discrimination mechanisms in the mammalian olfactory system using a model nose. Nature 1982, 299, 352-355.

(10) Yan, L.; Liu, J.; Jiang, S.; Wu, C.; Gao, K. The Regular Interaction Pattern among Odorants of the Same Type and Its Application in Odor Intensity Assessment. Sensors 2017, 17, 1624.

(11) Szulczyński, B.; Namieśnik, J.; Gȩbicki, J. Determination of Odour Interactions of Three-Component Gas Mixtures Using an Electronic Nose. Sensors 2017, 17, 2380.

(12) Hudon, G.; Guy, C.; Hermia, J. Measurement of Odor Intensity by an Electronic Nose. J. Air Waste Manage. Assoc. 2000, 50, 17501758.

(13) Vempatapu, B. P.; Kanaujia, P. K. Monitoring petroleum fuel adulteration: A review of analytical methods. TrAC Trends in Anal. Chem. 2017, 92, 1-11.

(14) Babu, V.; Krishna, R.; Mani, N. Review on the Detection of Adulteration in Fuels through Computational Techniques. Mater. Today: Proc. 2017, 4, 1723-1729.
(15) Kanat'eva, A. Y.; Dianov, M. E.; Kurganov, A. A. Identification of oxygen-containing compounds in gasoline by two-dimensional gas chromatography with mass spectrometric detection. Pet. Chem. 2012, 52, 356-361.

(16) Doble, P.; Sandercock, M.; Du Pasquier, E.; Petocz, P.; Roux, C.; Dawson, M. Classification. of premium and regular gasoline by gas chromatography/mass spectrometry, principal component analysis and artificial neural networks. Forensic Sci. Int. 2003, 132, 26-39.

(17) Lutz, O. M. D.; Bonn, G. K.; Rode, B. M.; Huck, C. W. Reproducible quantification of ethanol in gasoline via a customized mobile near-infrared spectrometer. Anal. Chim. Acta 2014, 826, 61-68.

(18) Bao, X.; Dai, L. Partial least squares with outlier detection in spectral analysis: A tool to predict gasoline properties. Fuel 2009, 88, $1216-1222$.

(19) Klingbeil, A. E.; Jeffries, J. B.; Hanson, R. K. Temperature- and composition-dependent mid-infrared absorption spectrum of gas-phase gasoline: Model and measurements. Fuel 2008, 87, 3600-3609.

(20) Singer, B. C.; Harley, R. A.; Littlejohn, D.; Ho, J.; Vo, T. Scaling of infrared remote sensor hydrocarbon measurements for motor vehicle emission inventory calculations. Environ. Sci. Technol. 1998, 32, 32413248.

(21) Zhang, X.; Qi, X.; Zou, M.; Wu, J. Rapid detection of gasoline by a portable Raman spectrometer and chemometrics. J. Raman Spectrosc. 2012, 43, 1487-1491.

(22) Ye, Q.; Xu, Q.; Yu, Y.; Qu, R.; Fang, Z. Rapid and quantitative detection of ethanol proportion in ethanol-gasoline mixtures by Raman spectroscopy. Opt. Commun. 2009, 282, 3785-3788.

(23) Khijwania, S. K.; Tiwari, V. S.; Yueh, F.-Y.; Singh, J. P. A fiber optic Raman sensor for hydrocarbon detection. Sens. Actuator B-Chem. 2007, 125, 563-568.

(24) Baumbach, J. I.; Sielemann, S.; Xie, Z.; Schmidt, H. Detection of the gasoline components methyl tert-butyl ether, benzene, toluene, and $\mathrm{m}$-xylene using ion mobility spectrometers with a radioactive and UV ionization source. Anal. Chem. 2003, 75, 1483-1490.

(25) Hu, F.; Zhang, L.; Xu, X.; Wang, Y.; Zou, T.; Zhang, W. Study on split-ring-resonator based terahertz sensor and its application to the identification of product oil. Opt. Quant. Electron. 2015, 47, 28672879.

(26) Arik, E.; Altan, H.; Esenturk, O. Dielectric Properties of Ethanol and Gasoline Mixtures by Terahertz Spectroscopy and an Effective Method for Determination of Ethanol Content of Gasoline. J. Phys. Chem. A 2014, 118, 3081-3089.

(27) Li, J.; Tian, Z.; Chen, Y.; Cao, W.; Zeng, Z. Distinguishing octane grades in gasoline using terahertz metamaterials. Appl. Opt. 2012, 51, $3258-3262$

(28) Steers, D.; Gerrard, C.; Hirst, B.; Sibbett, W.; Padgett, M. J. Gasoline analysis and brand identification using a static Fouriertransform ultraviolet spectrometer. J. Optic. Pure Appl. Optic. 1999, 1, $680-684$.

(29) Feldhoff, R.; Bernadet, P.; Saby, C. A. Discrimination of diesel fuels with chemical sensors and mass spectrometry based electronic noses. Analyst 1999, 124, 1167-1173.

(30) McCarrick, C. W.; Ohmer, D. T.; Gilliland, L. A.; Edwards, P. A.; Mayfield, H. T. Fuel identification by neural network analysis of the response of vapor-sensitive sensor arrays. Anal. Chem. 1996, 68, 42644269.

(31) Lauf, R. J.; Hoffheins, B. S. Analysis of liquid fuels using a gas sensor array. Fuel 1991, 70, 935-940.

(32) Wiziack, N. K. L.; Catini, A.; Santonico, M.; D’Amico, A.; Paolesse, R.; Paterno, L. G.; Fonseca, F. J.; Di Natale, C. A sensor array based on mass and capacitance transducers for the detection of adulterated gasolines. Sens. Actuator B-Chem. 2009, 140, 508-513.

(33) Cooper, J. S.; Raguse, B.; Chow, E.; Hubble, L.; Mueller, K.-H.; Wieczorek, L. Gold Nanoparticle Chemiresistor Sensor Array that Differentiates between Hydrocarbon Fuels Dissolved in Artificial Seawater. Anal. Chem. 2010, 82, 3788-3795.

(34) Eaidkong, T.; Mungkarndee, R.; Phollookin, C.; Tumcharern, G.; Sukwattanasinitt, M.; Wacharasindhu, S. Polydiacetylene paper-based 
colorimetric sensor array for vapor phase detection and identification of volatile organic compounds. J. Mater. Chem. 2012, 22, 5970-5977.

(35) Li, Z.; Jang, M.; Askim, J. R.; Suslick, K. S. Identification of accelerants, fuels and post-combustion residues using a colorimetric sensor array. Analyst 2015, 140, 5929-5935.

(36) Majhi, A.; Kukerti, V. S.; Sharma, Y. K.; Khanna, R.; Datta, A. The Detection of Kerosene as an Adulterant in Gasoline. Pet. Sci. Technol. 2012, 30, 271-277.

(37) Haddad, R.; Regiani, T.; Klitzke, C. F.; Sanvido, G. B.; Corilo, Y. E.; Augusti, D. V.; Pasa, V. M. D.; Pereira, R. C. C.; Romao, W.; Vaz, B. G.; Augusti, R.; Eberlin, M. N. Gasoline, Kerosene, and Diesel Fingerprinting via Polar Markers. Energy Fuels 2012, 26, 3542-3547.

(38) Shiba, K.; Tamura, R.; Sugiyama, T.; Kameyama, Y.; Koda, K.; Sakon, E.; Minami, K.; Ngo, H. T.; Imamura, G.; Tsuda, K.; Yoshikawa, G. Functional Nanoparticles-Coated Nanomechanical Sensor Arrays for Machine Learning-Based Quantitative Odor Analysis. ACS Sens. 2018, 3, 1592-1600.

(39) Shiba, K.; Tamura, R.; Imamura, G.; Yoshikawa, G. Data-driven nanomechanical sensing: specific information extraction from a complex system. Sci. Rep. 2017, 7, 3661.

(40) Yoshikawa, G.; Akiyama, T.; Gautsch, S.; Vettiger, P.; Rohrer, H. Nanomechanical Membrane-type Surface Stress Sensor. Nano Lett. 2011, 11, 1044-1048.

(41) Guerrero, R.; Nguyen, F.; Yoshikawa, G. Real-time gas identification on mobile platforms using a nanomechanical membrane-type surface stress sensor. EPJ Techn Instrum 2014, 1, 1-5.

(42) Yoshikawa, G.; Akiyama, T.; Loizeau, F.; Shiba, K.; Gautsch, S.; Nakayama, T.; Vettiger, P.; de Rooij, N. F.; Aono, M. Two Dimensional Array of Piezoresistive Nanomechanical Membrane-Type Surface Stress Sensor(MSS) with Improved Sensitivity. Sensors 2012, 12, $15873-15887$.

(43) Shiba, K.; Sugiyama, T.; Takei, T.; Yoshikawa, G. Controlled growth of silica-titania hybrid functional nanoparticles through a multistep microfluidic approach. Chem. Commun. 2015, 51, 1585415857.

(44) Loizeau, F.; Akiyama, T.; Gautsch, S.; Vettiger, P.; Yoshikawa, G.; de Rooij, N. F. Comparing membrane- and cantilever-based surface stress sensors for reproducibility. Sens. Actuators, A 2015, 228, 9-15.

(45) Chatterjee, S.; Castro, M.; Feller, J. F. An e-nose made of carbon nanotube based quantum resistive sensors for the detection of eighteen polar/nonpolar VOC biomarkers of lung cancer. J. Mater. Chem. B 2013, 1, 4563-4575.

(46) Bietsch, A.; Zhang, J.; Hegner, M.; Lang, H. P.; Gerber, C. Rapid functionalization of cantilever array sensors by inkjet printing. Nanotechnology 2004, 15, 873-880.

(47) Freund, M. S.; Lewis, N. S. A chemically diverse conducting polymer-based "electronic nose". Proc. Natl. Acad. Sci. U.S.A. 1995, 92, $2652-2656$.

(48) Minami, K.; Shiba, K.; Yoshikawa, G. Discrimination of structurally similar odorous molecules with various concentrations by using a nanomechanical sensor. Anal. Methods 2018, 10, 3720-3726.

(49) Zeng, H.; Zou, F.; Lehne, E.; Zuo, J. Y.; Zhang, D. Gas Chromatograph Applications in Petroleum Hydrocarbon Fluids. Adv. Gas Chromatogr. 2012, 17, 363-388.

(50) Smith, L. L.; Strickland, J. R. Improved GC/MS Method for Quantitation of n-Alkanes in Plant and Fecal Material. J. Agric. Food Chem. 2007, 55, 7301-7307.

(51) Wenzel, M. J.; Josse, F.; Heinrich, S. M.; Yaz, E.; Datskos, P. G. Sorption-induced static bending of microcantilevers coated with viscoelastic material. J. Appl. Phys. 2008, 103, No. 064913.

(52) Imamura, G.; Shiba, K.; Yoshikawa, G.; Washio, T. Analysis of nanomechanical sensing signals; physical parameter estimation for gas identification. AIP Adv. 2018, 8, No. 075007.

(53) Yoshikawa, G. Mechanical analysis and optimization of a microcantilever sensor coated with a solid receptor film. Appl. Phys. Lett. 2011, 98, 173502.

(54) Doleman, B. J.; Severin, E. J.; Lewis, N. S. Trends in odor intensity for human and electronic noses: Relative roles of odorant vapor pressure vs. molecularly specific odorant binding. Proc. Natl. Acad. Sci. U. S. A. 1998, 95, 5442-5447.

(55) Ichikawa, M.; Nonaka, N.; Takada, I.; Ishimori, S. Mass Spectrometric Analysis for Distinction between Regular and Premium Motor Gasolines. Anal. Sci. 1993, 9, 261-266.

(56) Sasidharan, M.; Zenibana, H.; Nandi, M.; Bhaumik, A.; Nakashima, K. Synthesis of mesoporous hollow silica nanospheres using polymeric micelles as template and their application as a drugdelivery carrier. Dalton Trans. 2013, 42, 13381-13389.

(57) Ide, Y.; Ogawa, M. Interlayer modification of a layered titanate with two kinds of organic functional units for molecule-specific adsorption. Angew. Chem., Int. Ed. 2007, 46, 8449-8451.

(58) Speller, N. C.; Siraj, N.; Regmi, B. P.; Marzoughi, H.; Neal, C.; Warner, I. M. Rational Design of QCM-D Virtual Sensor Arrays Based on Film Thickness, Viscoelasticity, and Harmonics for Vapor Discrimination. Anal. Chem. 2015, 87, 5156-5166. 